\title{
Capacity and SER Analysis of MIMO Beamforming with MRC
}

\author{
Matthew R. McKay \\ Telecommunications Lab. \\ School of Elec. and Info. Engineering \\ University of Sydney, Australia \\ and also the ICT Centre, CSIRO, Australia \\ mckay@ee.usyd.edu.au
}

\author{
Iain B. Collings \\ Wireless Technologies Lab. \\ ICT Centre \\ CSIRO \\ Sydney, Australia \\ Iain.Collings@csiro.au
}

\author{
Peter J. Smith \\ Dept. of Elec. and Comp. Engineering \\ University of Canterbury \\ Christchurch, New Zealand \\ p.smith@elec.canterbury.ac.nz
}

\begin{abstract}
We derive closed-form expressions for the ergodic capacity and symbol error rate (SER) of MIMO beamforming with maximum ratio combining (MRC) receivers in uncorrelated and semi-correlated Rayleigh channels. Our results are exact, finite expressions, applying for arbitrary numbers of antennas, and all SNRs. Based on the analytical results, we examine the effect of spatial correlation on the capacity and SER.
\end{abstract}

Index Terms-Beamforming, MIMO, capacity, channel correlation

\section{INTRODUCTION}

Multiple-input multiple-output (MIMO) transmit beamforming (BF) (also known as maximum-ratio transmission [1], MIMO maximum-ratio combining (MRC) [2-4], and transmitreceive diversity [5]) exploits channel knowledge at both the transmitter and receiver to help mitigate the severe effects of fading through diversity. MIMO-BF systems operate by appropriately weighting (or steering) the transmitted and received signals, such that the instantaneous signal to noise ratio (SNR) at the output of the receiver combiner is maximized.

Recently, various authors have examined the error performance of MIMO-BF in uncorrelated Rayleigh fading channels. In [5,6], systems were considered employing $M$-PSK/QAM modulation or binary signalling. Some symbol error rate (SER) expressions were presented in terms of unknown coefficients which required numerical evaluation via a symbolic software package. In [2,6-9], expressions were presented which did not involve these unknown coefficients. However, the results in $[2,6-8]$ were restricted to cases where there are only two transmit or receive antennas; whereas the results in [9] involved infinite summations over partitions of numbers, as well as zonal polynomials and hypergeometric coefficients, making them unsuitable for efficient numerical evaluation. For semi-correlated Rayleigh channels, SER results were presented in [4], however these expressions were not in closed-form.

Considering ergodic capacity, to our knowledge, the only analytical results for MIMO-BF were recently presented in [3], for uncorrelated Rayleigh channels. Their results were again expressed in terms of unknown coefficients (as for the SER results in $[5,6]$ ), and a numerical search algorithm was proposed.

In this paper, we derive closed-form expressions for both the ergodic capacity and SER of MIMO-BF, in both uncorrelated and semi-correlated Rayleigh channels. Our results are exact, finite expressions, which apply for arbitrary numbers of transmit and receive antennas and all SNRs, and do not contain coefficients requiring numerical evaluation. The key to our closed-form results is that we derive exact expressions for the probability density function (p.d.f.) and moment generating function (m.g.f.) of the largest eigenvalue of complex Wishart and complex correlated-Wishart matrices. For both ergodic capacity and SER, we use these results to present general expressions, as well as simplified expressions for cases when there are only two transmit or receive antennas. We note that, although the expressions in this paper may appear complicated, they are all essentially finite summations of functions which can be easily and efficiently evaluated.

Finally, based on the analytical results, we examine the effect of spatial correlation on the ergodic capacity and SER. We find that capacity increases with the correlation, whereas the SER will increase or decrease, depending on the SNR.

\section{MIMO BEAMFORMING SySTEM MODEL}

Consider a $N_{t} \times N_{r}$ MIMO-BF system, where the $N_{r} \times 1$ received signal vector is

$$
\mathbf{r}=\sqrt{\bar{\gamma}} \mathbf{H} \mathbf{w} x+\mathbf{n}
$$

where $x$ is the transmitted symbol with $E\left[|x|^{2}\right]=1$, w is the beamforming vector (specified below) with $E\left[\|\mathbf{w}\|^{2}\right]=$ $1, \mathbf{n}$ is noise $\sim \mathcal{C N}\left(\mathbf{0}, \mathbf{I}_{N_{r}}\right)$, and $\bar{\gamma}$ is the signal to noise ratio (SNR). Also, $\mathbf{H}$ is the $N_{r} \times N_{t}$ channel matrix, assumed to be flat spatially-correlated Rayleigh fading. Assuming the well-known Kronecker correlation structure [10-13] we can decompose $\mathbf{H}$ as

$$
\mathbf{H}=\mathbf{R}^{\frac{1}{2}} \mathbf{H}_{w} \mathbf{S}^{\frac{1}{2}}
$$

where $\mathbf{R}$ and $\mathbf{S}$ are the receive and transmit correlation matrices respectively, satisfying $\operatorname{tr}(\mathbf{R})=N_{r}$ and $\operatorname{tr}(\mathbf{S})=N_{t}$, and the elements of $\mathbf{H}_{w}$ are i.i.d. $\mathcal{C N}(0,1)$.

The receiver employs the principle of MRC to give

$$
\mathbf{z}=\sqrt{\bar{\gamma}} \mathbf{w}^{\dagger} \mathbf{H}^{\dagger} \mathbf{H} \mathbf{w} x+\mathbf{w}^{\dagger} \mathbf{H}^{\dagger} \mathbf{n} .
$$

Therefore, the SNR at the output of the combiner is

$$
\gamma=\bar{\gamma} \mathbf{w}^{\dagger} \mathbf{H}^{\dagger} \mathbf{H} \mathbf{w} \text {. }
$$

The $\mathrm{BF}$ vector $\mathbf{w}$ is chosen to maximize this instantaneous SNR, thereby minimizing the error probability. Based on this criterion, it is well known that the optimum $\mathrm{BF}$ vector $\mathbf{w}_{\mathrm{opt}}$ 


$$
\mathcal{M}_{\lambda_{\max }}(s)=1+\sum_{\ell=1}^{n} \sum_{\left\{\underline{\beta}_{\ell}\right\}} \sum_{\{\underline{\alpha}\}} \frac{(-1)^{\ell+\operatorname{per}(\underline{\alpha})} \Gamma_{m}(n, \underline{\alpha})}{\Gamma_{n}(n) \Gamma_{m}(n)} \sum_{k=0}^{K\left(\underline{\alpha}, \underline{\beta}_{\ell}\right)} \sum_{\left\{k_{1}, \ldots, k_{\ell}\right\}}\left(\begin{array}{c}
k \\
k_{1}, \ldots, k_{\ell}
\end{array}\right)(-1)^{k} \frac{s}{(s-\ell)^{k+1}}
$$

$$
\tilde{\mathcal{M}}_{\lambda_{\max }}(s)=1+\sum_{\ell=1}^{n} \sum_{\left\{\underline{\beta}_{\ell}\right\}} \sum_{\{\underline{\alpha}\}} \frac{(-1)^{\ell+\operatorname{per}(\underline{\alpha})} \mathcal{P}(\underline{\alpha})}{\operatorname{det}(\boldsymbol{\Sigma})^{\tau} V(\boldsymbol{\Sigma})} \sum_{k=0}^{\tilde{K}\left(\underline{\alpha}, \underline{\beta}_{\ell}\right)} \sum_{\left\{\tilde{k}_{1}, \ldots, \tilde{k}_{\ell}\right\}} \frac{\left(\begin{array}{c}
\tilde{k}_{1}, \ldots, \tilde{k}_{\ell} \\
\tilde{k}
\end{array}\right)(-1)^{\tilde{k}}}{\prod_{i=1}^{\ell} \sigma_{\beta_{i}}^{\tilde{k}_{i}}} \frac{s}{\left(s-\sum_{i=1}^{\ell} \sigma_{\beta_{i}}^{-1}\right)^{\tilde{k}+1}}
$$

is the eigenvector corresponding to the maximum eigenvalue $\lambda_{\max }$ of $\mathbf{H}^{\dagger} \mathbf{H}$. In this case, the beamformed SNR (4) becomes

$$
\gamma=\bar{\gamma} \mathbf{w}_{\mathrm{opt}}^{\dagger} \mathbf{H}^{\dagger} \mathbf{H} \mathbf{w}_{\mathrm{opt}}=\bar{\gamma} \lambda_{\max } .
$$

Clearly the performance of MIMO-BF depends directly on the statistical properties of $\lambda_{\max }$, which in turn depends on the fading scenario. For the case of uncorrelated Rayleigh fading we have $\mathbf{R}=\mathbf{I}_{N_{r}}$ and $\mathbf{S}=\mathbf{I}_{N_{t}}$, and $\lambda_{\max }$ has the same distribution as the maximum eigenvalue of a complex Wishart matrix $\mathbf{W} \sim \mathcal{W}_{n}\left(m, \mathbf{I}_{n}\right)$ (see $[5,12,13]$ for more details), where $n=\min \left(N_{r}, N_{t}\right)$ and $m=\max \left(N_{r}, N_{t}\right)$.

For semi-correlated channels with $n \times n$ correlation matrix

$$
\boldsymbol{\Sigma}= \begin{cases}\mathbf{R} & , N_{r} \leq N_{t} \\ \mathbf{S} & , N_{r}>N_{t}\end{cases}
$$

(i.e. correlation occurring at the end with the least antennas) $\lambda_{\max }$ has the same distribution as the maximum eigenvalue of a complex correlated Wishart matrix $\mathbf{W} \sim \mathcal{W}_{n}(m, \boldsymbol{\Sigma})$. For semi-correlation at the end with the most antennas, $\lambda_{\max }$ is distributed as the maximum eigenvalue of a complex correlated pseudo-Wishart matrix.

Due to space limitations, we restrict ourselves to Wishart and correlated-Wishart scenarios in this paper. In each case, for the error analysis we will require the moment generating function (m.g.f.) of the maximum eigenvalue, which we now consider.

\section{Moment Generating Function of the Maximum EIGENVALUE OF COMPLEX Wishart Matrices}

Theorem 1: Let $\mathbf{W} \sim \mathcal{W}_{n}\left(m, \mathbf{I}_{n}\right)$, where $n \leq m$, and define $\tau=m-n$. Then the m.g.f. of the maximum eigenvalue $\lambda_{\max }$ of $\mathbf{W}$ is given by (6) at the top of the page, where

$$
\Gamma_{m}(n)=\prod_{i=1}^{n} \Gamma(m-i+1),
$$

$\{\underline{\alpha}\}$ is the set of all permutations $\underline{\alpha}=\{\alpha(1), \ldots, \alpha(n)\}$ of $\mathcal{M} \triangleq\{1, \ldots, n\},(-1)^{\operatorname{per}(\underline{\alpha})}$ is the sign of the permutation; $\{\underline{\beta}\}$ is the collection of all subsets $\underline{\beta}_{\ell}=\left\{\beta_{1}, \ldots, \beta_{\ell}\right\}$ of $\mathcal{M}$, with $\beta_{1}<\ldots<\beta_{\ell}$; and

$$
\Gamma_{m}(n, \underline{\alpha})=\prod_{i=1}^{n} \Gamma(\tau+i+\alpha(i)-1) .
$$

Also,

$$
K\left(\underline{\alpha}, \underline{\beta}_{\ell}\right)=\sum_{i=1}^{\ell}\left(g\left(\beta_{i}, \alpha\left(\beta_{i}\right)\right)-1\right)
$$

where $g(i, j)=\tau+i+j-1$, and the last (finite) sum is over all partitions $\left\{k_{1}, \ldots, k_{\ell}\right\}$ of $k$ (i.e. $\sum_{i=1}^{\ell} k_{i}=k$ ) satisfying $0 \leq k_{i}<g\left(\beta_{i}, \alpha\left(\beta_{i}\right)\right), \forall i=1, \ldots, \ell ;$ and

$$
\left(\begin{array}{c}
k \\
k_{1}, \ldots, k_{\ell}
\end{array}\right)=\frac{k !}{k_{1} ! \ldots k_{\ell} !}
$$

is the multinomial coefficient.

Proof: See Appendix 1.

The following theorem is a more general case of Theorem 1. Note that, although the theorem can be shown to reduce to Theorem 1 for the special case $\Sigma=\mathbf{I}_{n}$, the reduction is not direct and requires limits and significant combinatoric manipulation.

Theorem 2: Let $\mathbf{W} \sim \mathcal{W}_{n}(m, \boldsymbol{\Sigma})$, where $n \leq m$, and $\boldsymbol{\Sigma}$ is an $n \times n$ Hermitian positive definite matrix with eigenvalues $\sigma_{1}, \ldots, \sigma_{n}$. Then the m.g.f. of the maximum eigenvalue $\lambda_{\max }$ of $\mathbf{W}$ is given by (11) at the top of the page, where

$$
V(\boldsymbol{\Sigma})=\prod_{i<j}\left(\sigma_{i}-\sigma_{j}\right)
$$

is a Vandermonde determinant, $\{\underline{\alpha}\}$ and $\left\{\underline{\beta}_{\ell}\right\}$ are defined as in Theorem 1, and

$$
\mathcal{P}(\underline{\alpha})=\prod_{i=1}^{n} \sigma_{i}^{m-\alpha(i)} .
$$

Also,

$$
\tilde{K}\left(\underline{\alpha}, \underline{\beta}_{\ell}\right)=\ell m-\sum_{i=1}^{\ell} \alpha\left(\beta_{i}\right)
$$

and the last (finite) sum is over all partitions $\left\{\tilde{k}_{1}, \ldots, \tilde{k}_{\ell}\right\}$ of $\tilde{k}$ (i.e. $\sum_{i=1}^{\ell} \tilde{k}_{i}=\tilde{k}$ ) satisfying $0 \leq \tilde{k}_{i} \leq m-\alpha\left(\beta_{i}\right), \forall i=$ $1, \ldots, \ell$.

Proof: The proof follows similar steps to the proof of Theorem 1 in Appendix I, however starting with the c.d.f. of $\lambda_{\max }$ in this semi-correlated case, given in [11, Theor. 4].

\section{ERGODIC CAPACITY}

This section considers the ergodic capacity (in $\mathrm{b} / \mathrm{s} / \mathrm{Hz}$ ) of MIMO-BF in uncorrelated and semi-correlated Rayleigh fading channels, which is given by the following expression

$$
C=E_{\lambda_{\max }}\left[\log _{2}\left(1+\lambda_{\max } \bar{\gamma}\right)\right]
$$




$$
C=\log _{2}(e) \sum_{\ell=1}^{n} \sum_{\left\{\underline{\beta}_{\ell}\right\}} \sum_{\{\underline{\alpha}\}} \frac{(-1)^{\ell+\operatorname{per}(\underline{\alpha})+1} \Gamma_{m}(n, \underline{\alpha})}{\Gamma_{n}(n) \Gamma_{m}(n)} \sum_{k=0}^{K\left(\underline{\alpha}, \underline{\beta}_{\ell}\right)} \sum_{\left\{k_{1}, \ldots, k_{\ell}\right\}} \frac{\left(\begin{array}{c}
k \\
k_{1}, \ldots, k_{\ell}
\end{array}\right)}{\ell^{k}} \mathrm{E}_{k+1}(\ell / \bar{\gamma}) e^{\ell / \bar{\gamma}}
$$

$$
\tilde{C}=\log _{2}(e) \sum_{\ell=1}^{n} \sum_{\left\{\underline{\beta}_{\ell}\right\}} \sum_{\{\underline{\alpha}\}} \frac{(-1)^{\ell+\operatorname{per}(\underline{\alpha})+1} \mathcal{P}(\underline{\alpha})}{\operatorname{det}(\boldsymbol{\Sigma})^{\tau} V(\boldsymbol{\Sigma})} \sum_{\tilde{k}=0}^{\tilde{K}\left(\underline{\alpha}, \underline{\beta}_{\ell}\right)} \sum_{\left\{\tilde{k}_{1}, \ldots, \tilde{k}_{\ell}\right\}} \frac{\left(\begin{array}{c}
\tilde{k}_{1}, \ldots, \tilde{k}_{\ell} \\
\tilde{k}^{\prime}
\end{array}\right.}{\left(\sum_{i=1}^{\ell} \sigma_{\beta_{i}}^{-1}\right)^{\tilde{k}}} \frac{e^{\sum_{i=1}^{\ell} \sigma_{\beta_{i}}^{-1} / \bar{\gamma}}}{\prod_{i=1}^{\ell} \sigma_{\beta_{i}}^{\tilde{k}_{i}}} \mathrm{E}_{\tilde{k}+1}\left(\frac{\sum_{i=1}^{\ell} \sigma_{\beta_{i}}^{-1}}{\bar{\gamma}}\right)
$$

\section{A. Uncorrelated Rayleigh Fading}

To evaluate the capacity in this case we use (35) in Appendix I to write

$$
\begin{aligned}
C=\int_{0}^{\infty} \log _{2}(1+x \bar{\gamma}) f_{\lambda_{\max }}(x) \mathrm{d} x \\
=\sum_{\ell=1}^{n}(-1)^{\ell} \sum_{\left\{\underline{\beta}_{\ell}\right\}} \sum_{\{\underline{\alpha}\}} \frac{(-1)^{\operatorname{per}(\underline{\alpha})} \Gamma_{m}(n, \underline{\alpha})}{\Gamma_{n}(n) \Gamma_{m}(n)} \\
\times \sum_{k=0}^{K\left(\underline{\alpha}, \underline{\beta}_{\ell}\right)} \sum_{\left\{k_{1}, \ldots, k_{\ell}\right\}} \frac{\mathcal{J}}{\prod_{i=1}^{\ell} k_{i} !}
\end{aligned}
$$

where

$$
\mathcal{J}=\int_{0}^{\infty} e^{-x \ell}\left(k x^{k-1}-\ell x^{k}\right) \log _{2}(1+x \bar{\gamma}) \mathrm{d} x .
$$

To evaluate this integral we combine a result from [14, App. B] and [15, eq 6.5.9], to give the identity

$$
\int_{0}^{\infty} t^{n-1} \ln (1+t) e^{-\mu t} \mathrm{~d} t=\frac{\Gamma(n) e^{\mu}}{\mu^{n}} \sum_{r=1}^{n} \mathrm{E}_{r}(\mu)
$$

for $\mu>0, n=1,2, \ldots$, where $\mathrm{E}_{r}(\cdot)$ is the Exponential Integral. After a simple change of variables, we apply this identity to evaluate (17), and then perform some algebraic manipulations to obtain the final closed-form capacity expression in (19) at the top of the page.

As mentioned previously, although (19) may appear complicated, it essentially involves finite summations of functions which can be efficiently evaluated.

Special Case, $n=2$ : In this case (19) reduces to

$$
\begin{aligned}
C= & \log _{2}(e) \sum_{i=0}^{2} \frac{(m-1)_{i}\left(\begin{array}{l}
2 \\
i
\end{array}\right)(-1)^{i}}{(m-i+1)_{i-1}}\left(e^{1 / \bar{\gamma}} \sum_{t=1}^{m+i-1} \mathrm{E}_{t}(1 / \bar{\gamma})\right. \\
& \left.-e^{2 / \bar{\gamma}} \sum_{k=0}^{m+i-2} \frac{(m-i+1)_{k}}{2^{m-i+1+k} k !} \sum_{t=1}^{m-i+1+k} \mathrm{E}_{t}(2 / \bar{\gamma})\right)
\end{aligned}
$$

where $(\cdot)_{\ell}$ is the Pochhamer symbol, defined as $(a)_{\ell}=a(a+$ 1) $\cdots(a+\ell-1)$, with $a_{0}=1$.

\section{B. Semi-Correlated Rayleigh Fading}

For semi-correlated channels, we derive the capacity using a similar procedure as for the uncorrelated case above. Omitting details, the p.d.f. of $\lambda_{\max }$ obtained from the derivation of (11) is used to calculate the capacity expression given by (20) at the top of the page.

Once again, (20) essentially involves finite summations of functions which can be efficiently evaluated.

\section{Symbol ERror Probability}

We now derive the SEP of MIMO-BF with uncoded $M$-PSK in uncorrelated and semi-correlated Rayleigh fading channels. It is well known that the SEP can be expressed as [16]

$$
P_{s}=\frac{1}{\pi} \int_{0}^{\left(\frac{M-1}{M}\right) \pi} M_{\gamma}\left(-\frac{g_{\mathrm{PSK}}}{\sin ^{2}(\theta)}\right) \mathrm{d} \theta
$$

where $g_{\mathrm{PSK}}=\sin ^{2}\left(\frac{\pi}{M}\right)$. From (5), this can be written as

$$
P_{s}=\frac{1}{\pi} \int_{0}^{\left(\frac{M-1}{M}\right) \pi} M_{\lambda_{\max }}\left(-\frac{g_{\mathrm{PSK}}}{\sin ^{2}(\theta)} \bar{\gamma}\right) \mathrm{d} \theta .
$$

Hence, we see that the error performance of MIMO-BF depends explicitly on the m.g.f.s presented in Section III.

\section{A. Uncorrelated Rayleigh Channels}

To calculate the SEP for uncorrelated Rayleigh channels, we use (6) in (22), and apply the integration identity [2]

$$
\begin{aligned}
& I(m, d, \theta) \triangleq \int_{0}^{\theta}\left(\frac{\sin ^{2}(\phi)}{\sin ^{2}(\phi)+d}\right)^{m} \mathrm{~d} \phi \\
& \quad=\frac{\left(\sin ^{2}(\theta)\right)^{m+\frac{1}{2}}}{(2 m+1) d^{m}} F_{1}\left(m+\frac{1}{2}, m, m+\frac{3}{2},-\frac{\sin ^{2}(\theta)}{d}, \sin ^{2}(\theta)\right)
\end{aligned}
$$

where $F_{1}(\cdot, \cdot, \cdot, \cdot, \cdot)$ is the Appell hypergeometric function [17], to obtain (23) at the top of the next page.

Note that an equivalent (yet less compact) finite expression can also be obtained in terms of elementary functions by calculating the integrals using the identity [18, eq. (77)].

Special Case, $n=2$ : In this case, the SER reduces to

$$
\begin{aligned}
P_{e} & =\frac{1}{\pi} \sum_{i=0}^{2} \frac{(m-1)_{i}\left(\begin{array}{l}
2 \\
i
\end{array}\right)(-1)^{i}}{(m-i+1)_{i-1}} \\
& \times\left(I\left(m+i-1, g_{\mathrm{psk}} \bar{\gamma}, \frac{(M-1) \pi}{M}\right)\right. \\
& \left.-\sum_{k=0}^{m+i-2} \frac{(m-i+1)_{k} I\left(m-i+1+k, \frac{g_{\mathrm{psk}} \bar{\gamma}}{2}, \frac{(M-1) \pi}{M}\right)}{2^{m-i+1+k} k !}\right)
\end{aligned}
$$

Note that this expression is simpler than the $n=2 M$ PSK SER result derived previously for uncorrelated Rayleigh channels in [2, Eq. 26].

\section{B. Semi-Correlated Rayleigh Channels}

For the semi-correlated case we use (11) in (22), and apply (38) from the proof of Theorem 1, to obtain the $M$-PSK SEP given by (24) at the top of the next page. 


$$
P_{s}=\frac{M-1}{M}+\sum_{\ell=1}^{n} \sum_{\left\{\underline{\beta}_{\ell}\right\}} \sum_{\{\underline{\alpha}\}} \frac{(-1)^{\ell+\operatorname{per}(\underline{\alpha})} \Gamma_{m}(n, \underline{\alpha})}{\Gamma_{n}(n) \Gamma_{m}(n)} \sum_{k=0}^{K\left(\underline{\alpha}, \underline{\beta}_{\ell}\right)} \sum_{\left\{k_{1}, \ldots, k_{\ell}\right\}} \frac{\left(\begin{array}{c}
k \\
k_{1}, \ldots, k_{\ell}
\end{array}\right)}{\ell^{k}} I_{k}\left(\frac{(M-1) \pi}{M}, \frac{g_{\mathrm{psk}} \bar{\gamma}}{\ell}\right)
$$

$$
\tilde{P}_{s}=\frac{M-1}{M}+\sum_{\ell=1}^{n} \sum_{\left\{\underline{\beta}_{\ell}\right\}} \sum_{\{\underline{\alpha}\}} \frac{(-1)^{\ell+\operatorname{per}(\underline{\alpha})} \mathcal{P}(\underline{\alpha})}{\operatorname{det}(\boldsymbol{\Sigma})^{\tau} V(\boldsymbol{\Sigma})} \sum_{\tilde{k}=0}^{\tilde{K}\left(\underline{\alpha}, \underline{\beta}_{\ell}\right)} \sum_{\left\{\tilde{k}_{1}, \ldots, \tilde{k}_{\ell}\right\}} \frac{\left(\begin{array}{c}
\tilde{k} \\
\tilde{k}_{1}, \ldots, \tilde{k}_{\ell}
\end{array}\right)}{\left(\sum_{i=1}^{\ell} \sigma_{\beta_{i}}^{-1}\right)^{\tilde{k}}} \frac{I_{\tilde{k}}\left(\frac{(M-1) \pi}{M}, \frac{g_{\mathrm{psk} \bar{\gamma}}}{\sum_{i=1}^{\ell} \sigma_{\beta_{i}}^{-1}}\right)}{\prod_{i=1}^{\ell} \sigma_{\beta_{i}}^{\tilde{k}_{i}}}
$$

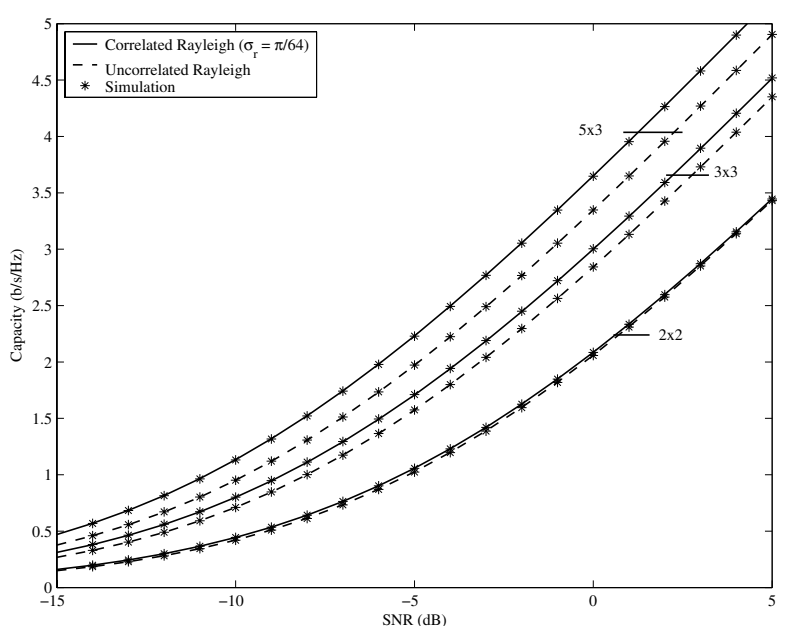

Fig. 1. Capacity of MIMO-BF in uncorrelated and semi-correlated Rayleigh channels, for various antenna configurations. Correlation parameters are $\theta_{r}=$ $\frac{\pi}{2}, d=1 / 2$, and $\sigma_{r}=\pi / 64$

\section{NumeriCAl RESUlts}

Fig. 1 shows the capacity curves for MIMO-BF with various antenna configurations in both uncorrelated and (receive) correlated Rayleigh channels. The correlated channels are constructed based on the model from [10], which include the parameters $\theta_{r}, d$, and $\sigma_{r}$, corresponding to the mean angle of arrival, relative antenna spacing, and cluster angle spread respectively. The uncorrelated curves are based on (19), and the correlated curves are based on (20). We see that the curves agree precisely with the Monte-Carlo simulated capacity. For the $2 \times 2$ case, we see that the uncorrelated and correlated capacity curves are almost identical, however for the higher number of antennas the capacity of the correlated channels is greater.

Fig. 2 shows the SEP with BPSK modulation. The antenna configurations and channel scenarios are the same as for Fig. 1. The uncorrelated curves are based on (23) and correlated curves are based on (24). In all cases the analytical results agree with the Monte-Carlo simulation results. Note that for each antenna configuration there is a cross-over SNR at which the correlated channels start performing worse than uncorrelated channels. This agrees with previous observations in [4]. We also see that as the antenna numbers increase, the performance in correlated channels becomes superior for a larger range of SNRs.

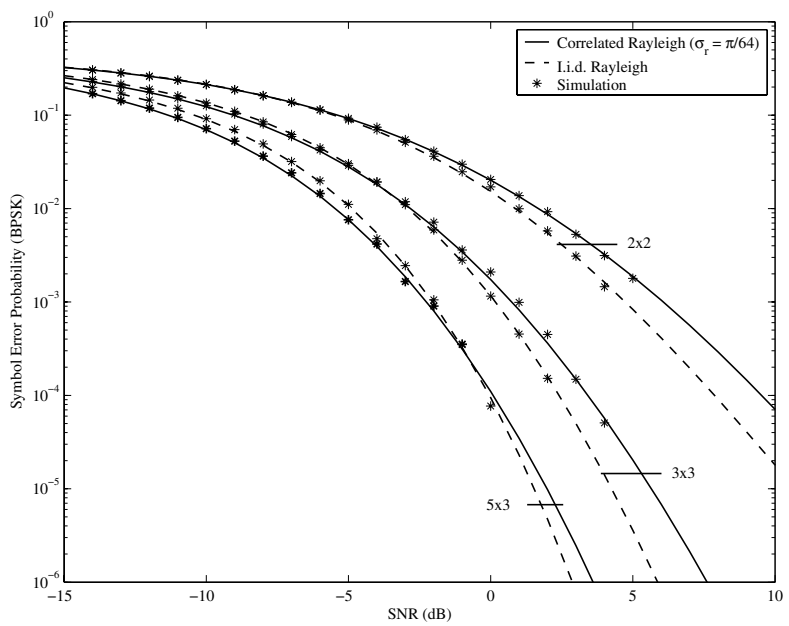

Fig. 2. SEP of MIMO-BF with BPSK in uncorrelated and semi-correlated Rayleigh channels, for various antenna configurations. Correlation parameters are $\theta_{r}=\frac{\pi}{2}, d=1 / 2$, and $\sigma_{r}=\pi / 64$

\section{CONCLUSION}

We have presented exact closed-form SER and capacity expressions for MIMO-BF with MRC receivers in uncorrelated and semi-correlated Rayleigh channels. Based on the analytical results, we have examined the effect of spatial correlation on the SER and capacity of MIMO-BF systems.

\section{APPENDIX I \\ PROOF OF THEOREM 1}

Proof: We start with the c.d.f. of $\lambda_{\max }$, which was previously derived in [5] as

$$
P\left(\lambda_{\max }<x\right)=\frac{\operatorname{det}(\boldsymbol{\Xi}(x))}{\Gamma_{n}(n) \Gamma_{m}(n)}
$$

where $\boldsymbol{\Xi}(x)$ is an $n \times n$ matrix with $(i, j)$ th entry

$$
\mathbf{\Xi}_{i j}(x)=\gamma(g(i, j), x)
$$

where $\gamma(\cdot)$ is the incomplete gamma function, defined as [17]

$$
\gamma(\ell, x)=\Gamma(\ell)\left(1-e^{-x} \sum_{k=0}^{\ell-1} \frac{x^{k}}{k !}\right)
$$

for integer $\ell$.

To evaluate the m.g.f., we expand the determinant as follows

$$
\operatorname{det}(\boldsymbol{\Xi}(x))=\sum_{\{\underline{\alpha}\}}(-1)^{\operatorname{per}(\underline{\alpha})} \prod_{i=1}^{n} \gamma(g(i, \alpha(i)), x) .
$$


Now using (27) in (28), it can be shown that

$$
\operatorname{det}(\boldsymbol{\Xi}(x))=\sum_{\{\underline{\alpha}\}}(-1)^{\operatorname{per}(\underline{\alpha})} \Gamma_{m}(n, \underline{\alpha}) P_{\alpha}
$$

where

$$
P_{\alpha}=\prod_{i=1}^{n}\left(1-e^{-x} \sum_{k=0}^{g(i, \alpha(i))-1} \frac{x^{k}}{k !}\right) .
$$

Next we use generating function expansion [19]

$$
\prod_{k=1}^{N}\left(1-x_{k} z\right)=1+\sum_{k=1}^{N}(-1)^{k} \operatorname{tr}_{k}\left(x_{1}, \ldots, x_{N}\right) z^{k}
$$

where $\operatorname{tr}_{k}(\cdot)$ denotes the $k^{\text {th }}$ elementary symmetric function (e.s.f.), defined in [20], to obtain

$$
P_{\alpha}=1+\sum_{\ell=1}^{n}(-1)^{\ell} \operatorname{tr}_{\ell}\left(\left\{e^{-x} \sum_{k=0}^{g(i, \alpha(i))-1} \frac{x^{k}}{k !}\right\}_{i=1, \ldots, n}\right) \text {. }
$$

Now applying the e.s.f. definition [20] we can write

$$
\begin{aligned}
P_{\alpha}=1 & +\sum_{\ell=1}^{n}(-1)^{\ell} \sum_{\left\{\underline{\beta}_{\ell}\right\}} \prod_{i=1}^{\ell}\left(e^{-x} \sum_{k=0}^{g\left(\beta_{i}, \alpha\left(\beta_{i}\right)\right)-1} \frac{x^{k}}{k !}\right) \\
= & +\sum_{\ell=1}^{n}(-1)^{\ell} e^{-\ell x} \\
& \times \sum_{\left\{\underline{\beta}_{\ell}\right\}} \sum_{k_{1}=0}^{g\left(\beta_{1}, \alpha\left(\beta_{1}\right)\right)-1} \ldots \sum_{k_{\ell}=0}^{g\left(\beta_{\ell}, \alpha\left(\beta_{\ell}\right)\right)-1} \frac{x^{\sum_{i=1}^{\ell} k_{i}}}{\prod_{i=1}^{\ell} k_{i} !} \\
= & +\sum_{\ell=1}^{n}(-1)^{\ell} e^{-\ell x} \sum_{\left\{\underline{\beta}_{\ell}\right\}} \sum_{k=0}^{K\left(\underline{\alpha}_{\ell}\right)} \sum_{\left\{k_{1}, \ldots, k_{\ell}\right\}} \frac{x^{k}}{\prod_{i=1}^{\ell} k_{i} !} .
\end{aligned}
$$

We now evaluate the m.g.f. of $\lambda_{\max }$, defined as

$$
\mathcal{M}_{\lambda_{\max }}(s)=\int_{0}^{\infty} e^{s x} f_{\lambda_{\max }}(x) \mathrm{d} x
$$

where $f_{\lambda_{\max }}(\cdot)$ is the p.d.f. of $\lambda_{\max }$. We evaluate $f_{\lambda_{\max }}(\cdot)$ by combining (33), (29), and (25), and differentiating, to give

$$
\begin{aligned}
f_{\lambda_{\max }}(x)=\sum_{\ell=1}^{n}( & -1)^{\ell} e^{-\ell x} \sum_{\left\{\underline{\beta}_{\ell}\right\}\{\underline{\alpha}\}} \frac{(-1)^{\operatorname{per}(\underline{\alpha})} \Gamma_{m}(n, \underline{\alpha})}{\Gamma_{n}(n) \Gamma_{m}(n)} \\
& \times \sum_{k=0}^{K\left(\underline{\alpha}_{\ell}\right)} \sum_{\left\{k_{1}, \ldots, k_{\ell}\right\}} \frac{k x^{k-1}-\ell x^{k}}{\prod_{i=1}^{\ell} k_{i} !}
\end{aligned}
$$

Substituting (35) into (34) yields

$$
\begin{aligned}
M_{\lambda_{\max }}(s)=\sum_{\ell=1}^{n} \sum_{\left\{\underline{\beta}_{\ell}\right\}} \sum_{\{\underline{\alpha}\}} \frac{(-1)^{\ell+\operatorname{per}(\underline{\alpha})} \Gamma_{m}(n, \underline{\alpha})}{\Gamma_{n}(n) \Gamma_{m}(n)} \\
\times \sum_{k=0}^{K\left(\underline{\alpha}, \underline{\beta}_{\ell}\right)} \sum_{\left\{k_{1}, \ldots, k_{\ell}\right\}} \frac{\mathcal{I}}{\prod_{i=1}^{\ell} k_{i} !}
\end{aligned}
$$

where

$$
\mathcal{I}=\int_{0}^{\infty} e^{-(\ell-s) x}\left(k x^{k-1}-\ell x^{k}\right) \mathrm{d} x .
$$

Using the identity [17]

$$
\int_{0}^{\infty} x^{k} e^{-\mu x} \mathrm{~d} x=k ! \mu^{-(k+1)}, \quad k=0,1, \ldots
$$

and performing some basic manipulations, we obtain

$$
\mathcal{I}= \begin{cases}s /(s-\ell)-1 & , k=0 \\ (-1)^{k} k ! s /(s-\ell)^{k+1} & , k>0\end{cases}
$$

Finally, substituting (39) into (36), and simplifying using the property $M_{\lambda_{\max }}(0)=1$, yields the desired result.

\section{REFERENCES}

[1] T. K. Y. Lo, "Maximum ratio transmission," IEEE Trans. Commun. vol. 47, no. 10, pp. 1458-1461, Oct 1999

[2] M. Kang and M.-S. Alouini, "A comparative study on the performance of MIMO MRC with and without cochannel interference," IEEE Trans. Commun., vol. 52, no. 8, pp. 1417-1425, Aug 2004.

[3] A. Maaref and S. Aïssa, "Closed-form exprressions for the outage and ergodic Shannon capacity of MIMO MRC systems," IEEE Trans. Commun., vol. 53, no. 7, pp. 1092-1095, Jul 2005.

[4] A. Zanella, M. Chiani, and M. Z. Win, "Performance of MIMO MRC in correlated Rayleigh fading environments," in Proc. IEEE Veh. Tech. Conf. (VTC), vol. 3, Stockholm, Sweden, Jun 2005, pp. 1633-1637.

[5] P. A. Dighe, R. K. Mallik, and S. S. Jamuar, "Analysis of transmitreceive diversity in Rayleigh fading," IEEE Trans. Commun., vol. 51, no. 4, pp. 694-703, Apr 2003.

[6] Y. Chen and C. Tellambura, "Performance analysis of maximum ratio transmission with imperfect channel estimation," IEEE Commun. Lett., vol. 9, no. 4, pp. 322-324, Apr 2005.

[7] B. D. Rao and M. Yan, "Performance of maximal ratio transmission with two receive antennas," IEEE Trans. Commun., vol. 51, no. 6, pp. 894-895, Jun 2003.

[8] S. Zhou and G. Giannakis, "How accurate channel prediction needs to be for transmit-beamforming with adaptive modulation over Rayleigh MIMO channels," IEEE Trans. Wireless Commun., vol. 52, no. 4, pp. 1285-1294, Jul 2004.

[9] A. J. Grant, "Performance analysis of transmit beamforming," IEEE Trans. Commun., vol. 53, no. 4, pp. 738-744, Apr 2005.

[10] H. Bölcskei and A. J. Paulraj, "Performance of space-time codes in the presence of spatial fading correlation," in Proc. Asilomar Conf., Sept 2000, pp. 687-693.

[11] M. Kang and M.-S. Alouini, "Impact of correlation on the capacity of MIMO channels," in Proc. IEEE Int. Conf. on Commun. (ICC), Paris, France, Jun 2003, pp. 2623-2627.

[12] P. J. Smith, S. Roy, and M. Shafi, "Capacity of MIMO systems with semicorrelated flat fading," IEEE Trans. Inform. Theory, vol. 49, no. 10, pp. 2781-2788, Oct 2003.

[13] M. R. McKay and I. B. Collings, "General capacity bounds for spatially correlated Rician MIMO channels," IEEE Trans. Inform. Theory, vol. 51, pp. 3121-3145, Sep. 2005.

[14] M.-S. Alouini and A. J. Goldsmith, "Capacity of Rayleigh fading channels under different adaptive transmission and diversity-combining techniques," IEEE Trans. Veh. Technol., vol. 48, no. 4, pp. 1165-1181, Jul 1999.

[15] M. Abramowitz and I. A. Stegun, Handbook of Mathematical Functions with Formulas, Graphs, and Mathematical Tables, 9th ed. New York: Dover Publications, 1970.

[16] M. K. Simon and M.-S. Alouini, Digital Communication over Fading Channels: A Unified Approach to Performance Analysis. New York, NY: John Wiley and Sons, Inc., 2000.

[17] I. S. Gradshteyn and I. M. Ryzhik, Table of Integrals, Series, and Products, 6th ed. San Diego, CA: Academic, 2000.

[18] M.-S. Alouini and M. K. Simon, "An MGF-based performance analysis of generalized selection combining over Rayleigh fading channels," IEEE Trans. Commun., vol. 48, no. 3, pp. 401-415, Mar 2000.

[19] A. B. Balantekin, "Character expansions, Itzykson-Zuber intergrals, and the QCD partition function," Phys. Rev. D, vol. 62, pp. $085017(1)$ $085017(8)$, Sep 2000.

[20] A. W. Marshall and I. Olkin, Inequalities: Theory of Majorization and 